\title{
Decomposition matrices for $d$-Harish-Chandra series: the exceptional rank two cases
}

\author{
Maria Chlouveraki and Hyohe Miyachi
}

\begin{abstract}
We calculate all decomposition matrices of the cyclotomic Hecke algebras of the rank two exceptional complex reflection groups in characteristic zero. We prove the existence of canonical basic sets in the sense of Geck-Rouquier and show that all modular irreducible representations can be lifted to the ordinary ones.
\end{abstract}

\section{Introduction}

\subsection{Notation}

Throughout this paper, $W$ will be a complex reflection group and $K$ will be its field of definition, that is, the smallest field to which the traces of all the elements of $W$ belong. We denote by $\mu(K)$ the group of all roots of unity in the number field $K$. For any positive integer $d$, we set $\zeta_{d}:=\exp (2 \pi i / d)$ and we denote by $\Phi_{d}(x)$ the $d$ th cyclotomic polynomial over $\mathbb{Q}$. From now on, let $d$ be a positive integer and let $q$ be an indeterminate.

\subsection{Motivation from $d$-Harish-Chandra theory}

In [7], the cyclotomic Hecke algebra $\mathcal{H}_{q}(W)$ associated with the complex reflection group $W$ was introduced: it is defined over $\mathbb{C}\left[q, q^{-1}\right]$ as a quotient algebra of the group algebra of the braid group associated with $W$. The motivation for introducing this algebra is that $\mathcal{H}_{q}(W)$ should appear naturally as an endomorphism algebra of $R_{L}^{G}(\boldsymbol{\lambda})$, where $R_{L}^{G}$ is the DeligneLusztig twisted induction functor for the finite reductive group $G$ and the so-called $d$-cuspidal pair $(L, \boldsymbol{\lambda})$ (cf. [5]). The complex $R_{L}^{G}(\boldsymbol{\lambda})$ is a truncation of the one giving a so-called Broué abelian defect conjecture. In particular, if we take $d=1$ and $\boldsymbol{\lambda}=1$, this picture goes back to the classical one, where $R_{L}^{G}(\boldsymbol{\lambda})$ is the permutation module over the (finite) Borel subgroup of $G$ and the Hecke algebra is the classical Iwahori-Hecke algebra. Hence, $\mathcal{H}_{q}(W)$ should be treated as the endomorphism algebra of the $d$-analogue of the permutation module over the Borel subgroup (principal series modules), that is the $d$-Harish-Chandra series.

The important points for $\mathcal{H}_{q}(W)$ are that:

- this algebra is a flat deformation of the group algebra of $W$ (actually, for $q=\zeta_{d}, \mathcal{H}_{q}(W)$ specializes to the group algebra $\mathbb{C} W$ );

- the relations deforming the group algebra multiplication ('cyclotomic relations') could involve multiparameters;

- there should be some distinguished choices of those parameters that are matched up with the endomorphism ring of the complex that we have seen above;

- those special choices for parameters are not known in general ${ }^{\dagger}$.

Received 21 August 2010; revised 21 June 2011.

2000 Mathematics Subject Classification 20C08 (primary), 20G40, 20C20 (secondary).

$\dagger$ The authors are indebted to C. Bonnafé for informing them of this open problem. 


\subsection{Aim and results}

In this paper, we shall consider the asymptotic modular representation theory of the $d$-HarishChandra series in the case where the conjectural choices for the distinguished parameters are known and $W$ is an exceptional (irreducible) complex reflection group of rank two. Here, by 'asymptotic' we mean the modular representation theory in characteristic zero. The conjectural choices of the parameters by Broué and Malle (cf. $[4,23])$ are given in the following table ${ }^{\dagger}$.

\begin{tabular}{ccccc}
\hline$W$ & $G$ & $d$ & $\lambda$ & Parameters of $\mathcal{H}_{q}(W)$ \\
\hline$G_{4}$ & ${ }^{3} D_{4}$ & 3 & 1 & $1, q, q^{2}$ \\
$G_{5}$ & & & & \\
& $F_{4}$ & 3 & 1 & $1, q, q^{2} ; 1, q, q^{2}$ \\
& ${ }^{2} E_{6}$ & 3 & 1 & $1, q, q^{2} ; 1, q^{2}, q^{4}$ \\
& $E_{8}$ & 3 & ${ }^{3} D_{4}[-1]$ & $1, q, q^{2} ; 1, q^{4}, q^{8}$ \\
$G_{8}$ & ${ }^{2} F_{4}$ & $8^{\prime}$ & 1 & $1, \zeta_{8}^{3} q, \zeta_{8}^{5} q, q^{2}$ \\
& $F_{4}$ & 4 & 1 & $1, q,-q, q^{2}$ \\
& ${ }^{2} E_{6}$ & 4 & 1 & $1, q, q^{2}, q^{3}$ \\
& $E_{7}$ & 4 & $\phi_{2}^{3}$ & $1, q,-q,-q^{4}$ \\
& & 4 & $\phi_{11}^{3}$ & $1, q^{3},-q^{3},-q^{4}$ \\
& $E_{8}$ & 4 & $\phi_{3,1}$ & $1,-1,-q, q^{5}$ \\
& & 4 & $\phi_{123,013}$ & $1,-q^{4}, q^{5},-q^{5}$ \\
& & 4 & $\phi_{013,2}$ & $1,-q,-q^{4}, q^{5}$ \\
$G_{9}$ & $E_{8}$ & 8 & 1 & $1, q^{4} ; 1, q^{2}, q^{4}, q^{6}$ \\
$G_{10}$ & $E_{8}$ & 12 & 1 & $1,-q^{2}, q^{4} ; 1, q^{3},-q^{3}, q^{6}$ \\
$G_{12}$ & ${ }^{2} F_{4}$ & 4 & 1 & $1, q^{2}$ \\
$G_{16}$ & $E_{8}$ & 5 & 1 & $1, q, q^{2}, q^{3}, q^{4}$ \\
$G_{20}$ & $H_{4}$ & 3 & 1 & $1, q, q^{2}$ \\
$G_{22}$ & $H_{4}$ & 4 & 1 & $1, q^{2}$ \\
\hline & & & &
\end{tabular}

We will calculate the decomposition matrices for all of the above cases and show the existence of canonical basic sets, as defined by Geck and Rouquier. The existence of canonical basic sets has already been proved for all Weyl groups for all choices of parameters in characteristic zero (cf. $[10,15,16,19])$. In that sense, $d$-Harish-Chandra theory works as the ordinary HarishChandra theory. What is more, we will see that, for the exceptional complex reflection groups of rank two, all 'modular' irreducible representations are obtained as modular reductions of some 'ordinary' ones, which therefore comprise an optimal basic set.

\section{Preliminaries}

\subsection{Cyclotomic Hecke algebras}

Let $V$ be a finite-dimensional $K$-vector space such that $W \subset \mathrm{GL}(V)$ acts irreducibly on $V$. Let us denote by $\mathcal{A}$ the set of reflecting hyperplanes of $W$ in $V$ and set $\mathcal{M}:=\mathbb{C} \otimes V-\bigcup_{H \in \mathcal{A}} \mathbb{C} \otimes H$. For $x_{0} \in \mathcal{M}$, let $B=\Pi_{1}\left(\mathcal{M} / W, x_{0}\right)$ be the braid group associated with $W$.

\footnotetext{
${ }^{\dagger}$ Some cases are omitted, since they can obtained from others by replacing $q$ with $-q$. Moreover, we include the cases corresponding to $H_{4}$ for the same reasons that they are included in $[4,23]$.
} 
For every orbit $\mathcal{C}$ of $W$ on $\mathcal{A}$, we set $e_{\mathcal{C}}$ to be the common order of the subgroups $W_{H}$, where $H$ is any element of $\mathcal{C}$ and $W_{H}$ the subgroup formed by $\mathrm{id}_{V}$ and all the reflections fixing the hyperplane $H$.

We choose a set of indeterminates $\mathbf{u}=\left(u_{\mathcal{C}, j}\right)_{(\mathcal{C} \in \mathcal{A} / W)\left(0 \leqslant j \leqslant e_{\mathcal{C}}-1\right)}$ and we denote by $\mathbb{Z}\left[\mathbf{u}, \mathbf{u}^{-1}\right]$ the Laurent polynomial ring in all the indeterminates $\mathbf{u}$. We define the generic Hecke algebra $\mathcal{H}(W)$ of $W$ to be the quotient of the group algebra $\mathbb{Z}\left[\mathbf{u}, \mathbf{u}^{-1}\right] B$ by the ideal generated by the elements of the form

$$
\left(\mathbf{s}-u_{\mathcal{C}, 0}\right)\left(\mathbf{s}-u_{\mathcal{C}, 1}\right) \ldots\left(\mathbf{s}-u_{\mathcal{C}, e_{\mathcal{C}}-1}\right),
$$

where $\mathcal{C}$ runs over the set $\mathcal{A} / W$ and $\mathbf{s}$ runs over the set of monodromy generators around the images in $\mathcal{M} / W$ of the elements of the hyperplane orbit $\mathcal{C}^{\dagger}$.

Let $\zeta_{d} \in \mu(K)$. A $\zeta_{d}$-cyclotomic specialization is a morphism of $\mathbb{C}$-algebras of the form

$$
\begin{aligned}
\varphi: \mathbb{C}\left[\mathbf{u}, \mathbf{u}^{-1}\right] & \rightarrow \mathbb{C}\left[q, q^{-1}\right] \\
u_{\mathcal{C}, j} & \mapsto \zeta_{e_{\mathcal{C}}}^{j}\left(\zeta_{d}^{-1} q\right)^{n_{\mathcal{C}, j}}
\end{aligned}
$$

with $n_{\mathcal{C}, j} \in \mathbb{Z}$ for all $\mathcal{C}, j$. The algebra $\mathcal{H}_{\varphi}(W)$ obtained as the specialization of $\mathcal{H}(W)$ via the morphism $\varphi$ is a $\zeta_{d^{-}}$cyclotomic Hecke algebra. For $q=\zeta_{d}, \mathcal{H}_{\varphi}(W)$ specializes to the group algebra $\mathbb{C} W$. A cyclotomic Hecke algebra is a Hecke algebra that is $\zeta_{d}$-cyclotomic for some positive integer $d$.

Now, in the spirit of generalized Harish-Chandra theory, $\zeta_{d}$-cyclotomic Hecke algebras may correspond to $d$-Harish-Chandra series for some finite reductive group. The table of $\S 1.3$ gives us a list of all cyclotomic Hecke algebras associated with exceptional complex reflection groups of rank two that should appear as $d$-Harish-Chandra series for some finite reductive group $G$. Each one of these Hecke algebras is $\zeta_{d}$-cyclotomic for the corresponding $d$.

\subsection{Schur elements and generic degrees}

From now on, $\mathcal{H}_{q}$ will denote a cyclotomic Hecke algebra associated with the group $W$. Set $y^{|\mu(K)|}:=q$. The algebra $\mathbb{C}(y) \mathcal{H}_{q}:=\mathbb{C}(y) \otimes_{\mathbb{C}\left[q, q^{-1}\right]} \mathcal{H}_{q}$ is split semisimple [9, Proposition 4.3.4] and, by Tits's deformation theorem, there exists a bijection $\operatorname{Irr}\left(\mathbb{C}(y) \mathcal{H}_{q}\right) \rightarrow \operatorname{Irr}(W), \chi_{q} \mapsto \chi$.

Furthermore, it is conjectured that $\mathcal{H}_{q}$ has a symmetrizing form $t_{q}: \mathcal{H}_{q} \rightarrow \mathbb{C}\left[q, q^{-1}\right]$ (that is, a trace function such that the bilinear form $\left(h, h^{\prime}\right) \mapsto t_{q}\left(h h^{\prime}\right)$ is non-degenerate) with nice properties (cf. [6, Theorem 2.1]): for example, $t_{q}$ specializes to the canonical symmetrizing form on $\mathbb{C} W$ for $q=\zeta_{d}$. This conjecture has been verified for all but a finite number of cases (see [24] for the list of cases). In particular, it has been verified for all groups studied in this paper, except for $G_{16}, G_{20}$ and $G_{22}$.

Due to Geck [14], we know that

$$
t_{q}=\sum_{\chi \in \operatorname{Irr}(W)} \frac{1}{s_{\chi}} \chi_{q},
$$

where $s_{\chi}$ is the Schur element of $\mathcal{H}_{q}$ associated with $\chi \in \operatorname{Irr}(W)$. The Schur elements belong to $\mathbb{Z}_{K}\left[y, y^{-1}\right]$ and they are products of cyclotomic polynomials over $K$ (cf. [9, Proposition 4.3.5]). For all $\chi \in \operatorname{Irr}(W)$, we set

$$
a_{\chi}:=\frac{\operatorname{valuation}\left(s_{\chi}(y)\right)}{|\mu(K)|} .
$$

Finally, let $P(y) \in K\left[y, y^{-1}\right]$ be a product of cyclotomic polynomials over $K$ that is divisible by all Schur elements of $\mathcal{H}_{q}$. Following $[\mathbf{6}, \S 6 \mathrm{E}]$, we define the $P$-generic degree of $\mathcal{H}_{q}$ associated

\footnotetext{
${ }^{\dagger}$ The monodromy generators are the generators of the braid group $B$. For their definition, the reader may refer, for example, to $[\mathbf{9}, \S 4.1 .2]$.
} 
with $\chi \in \operatorname{Irr}(W)$ to be the quotient

$$
D_{\chi}^{P}:=\frac{P(y)}{s_{\chi}}
$$

If we take $P(y)$ to be the relative Poincaré polynomial of $\mathcal{H}_{q}$ (in the sense of Broué-MalleMichel $[6, \S 6 \mathrm{~A}])$, then $D_{\chi}^{P}$ is called simply the generic degree. If $\boldsymbol{\lambda}=1$ in the corresponding cuspidal pair, then the relative Poincaré polynomial is equal to the Schur element associated with the trivial character (up to multiplication by a power of $y$ ).

\subsection{Decomposition matrix and basic sets}

Let $\theta: \mathbb{C}\left[y, y^{-1}\right] \rightarrow \mathbb{C}$ be a ring homomorphism such that $\theta(q)=\xi \in \mathbb{C}^{\times}$. Considering $\mathbb{C}$ as a $\mathbb{C}\left[y, y^{-1}\right]$-module via $\theta$, we set $\mathcal{H}_{\xi}:=\mathbb{C} \otimes_{\mathbb{C}\left[y, y^{-1}\right]} \mathcal{H}_{q}$. We call $\theta$ a specialization of $\mathcal{H}_{q}$. Note that the algebra $\mathcal{H}_{\xi}$ is split.

Recall that there is a bijection $\operatorname{Irr}\left(\mathbb{C}(y) \mathcal{H}_{q}\right) \leftrightarrow \operatorname{Irr}(W)$. Let $R_{0}\left(\mathbb{C}(y) \mathcal{H}_{q}\right)$ (respectively $R_{0}\left(\mathcal{H}_{\xi}\right)$ ) denote the Grothendieck group of finitely generated $\mathbb{C}(y) \mathcal{H}_{q^{-}}$modules (respectively $\mathcal{H}_{\xi^{-}}$ modules). It is generated by the classes $[U]$ of the simple $\mathbb{C}(y) \mathcal{H}_{q}$-modules (respectively $\mathcal{H}_{\xi}$-modules) $U$. Following [17, Theorem 7.4.3], we obtain a well-defined decomposition map

$$
d^{\theta}: R_{0}\left(\mathbb{C}(y) \mathcal{H}_{q}\right) \rightarrow R_{0}\left(\mathcal{H}_{\xi}\right)
$$

with corresponding decomposition matrix $\left(d_{\chi, \phi}^{\theta}\right)_{\chi \in \operatorname{Irr}(W), \phi \in \operatorname{Irr}\left(\mathcal{H}_{\xi}\right)}$. The decomposition matrix records information about the irreducible representations of the specialized Hecke algebra $\mathcal{H}_{\xi}$ in terms of the irreducible representations of the split semisimple Hecke algebra $\mathbb{C}(y) \mathcal{H}_{q}$. In particular, it records how the irreducible representations of $\mathbb{C}(y) \mathcal{H}_{q}$ break into irreducible representations of $\mathcal{H}_{\xi}$.

If $\mathcal{H}_{\xi}$ is semisimple, then Tits's deformation theorem implies that there exists a canonical bijection between the set of irreducible characters of $\mathbb{C}(y) \mathcal{H}_{q}$ and the set of irreducible characters of $\mathcal{H}_{\xi}$. Hence, the corresponding decomposition matrix is the identity matrix and the simple modules of $\mathcal{H}_{\xi}$ are parametrized by $\operatorname{Irr}(W)$. However, what do we do when the algebra $\mathcal{H}_{\xi}$ is not semisimple?

The theory of basic sets gives standard ways to parametrize the simple modules for Hecke algebras. A basic set is a subset of $\operatorname{Irr}(W)$ that is in bijection with the set of irreducible characters of $\mathcal{H}_{\xi}$. We will be mostly interested in two kinds of basic sets: the 'canonical' basic sets (in the sense of Geck-Rouquier [19]) and the 'optimal' ones.

Definition 1. We say that $\mathcal{H}_{q}$ admits a canonical basic set $\mathcal{B}^{\text {can }} \subset \operatorname{Irr}(W)$ with respect to $\theta$ if and only if the following two conditions are satisfied:

(1) for all $\phi \in \operatorname{Irr}\left(\mathcal{H}_{\xi}\right)$, there exists $\chi_{\phi} \in \mathcal{B}^{\text {can }}$ such that

(a) $d_{\chi_{\phi}, \phi}^{\theta}=1$;

(b) if $d_{\psi, \phi}^{\theta} \neq 0$ for some $\psi \in \operatorname{Irr}(W)$, then either $\psi=\chi_{\phi}$ or $a_{\psi}>a_{\chi_{\phi}}{ }^{\dagger}$;

(2) the map $\operatorname{Irr}\left(\mathcal{H}_{\xi}\right) \mapsto \mathcal{B}^{\text {can }}, \phi \mapsto \chi_{\phi}$ is a bijection.

\footnotetext{
${ }^{\dagger}$ The original definition of the canonical basic set uses Lusztig's $a$-function, which is defined as the opposite of the $a$-function that we use here. In the case of classical Iwahori-Hecke algebras of Weyl groups, the original definition yields that the trivial character has minimal $a$-value in $\operatorname{Irr}(W)$ (which is equal to zero). However, this fact depends on the order in which we write down the parameters of our algebra. Therefore, in the cases that will be studied in this paper, since we copy the order of parameters from $[4,23]$, we prefer to use the $a$ function defined in $\S 2.1$, so that the trivial character has always minimal $a$-value. This choice does not affect the existence of canonical basic sets (see also [10]), but may affect the parametrization of the characters belonging to them.
} 
Hence, if $\mathcal{H}_{q}$ admits a canonical basic set, then we can reorder the rows of the decomposition matrix so that its upper part (consisting of the rows indexed by the elements of $\mathcal{B}^{\text {can }}$ ) is a unitriangular matrix.

The existence of canonical basic sets in characteristic zero has been proved for all choices of parameters for Weyl groups $[\mathbf{1 0}, \mathbf{1 5}, \mathbf{1 6}, \mathbf{1 9}]$, for the Ariki-Koike algebras $[\mathbf{1}, \mathbf{1 2}, \mathbf{1 9}, \mathbf{2 2}, \mathbf{2 7}]$ and for certain cyclotomic Hecke algebras associated with the groups $G(d e, e, r)[\mathbf{2 0}]$.

Definition 2. We say that $\mathcal{H}_{q}$ admits an optimal basic set $\mathcal{B}^{\text {opt }} \subset \operatorname{Irr}(W)$ with respect to $\theta$ if and only if the following two conditions are satisfied:

(1) for all $\phi \in \operatorname{Irr}\left(\mathcal{H}_{\xi}\right)$, there exists $\chi_{\phi} \in \mathcal{B}^{\text {opt }}$ such that

(a) $d_{\chi_{\phi}, \phi}^{\theta}=1$

(b) if $d_{\psi, \phi}^{\theta} \neq 0$ for some $\psi \in \operatorname{Irr}(W)$, then either $\psi=\chi_{\phi}$ or $\psi \notin \mathcal{B}^{\text {opt }}$;

(2) the map $\operatorname{Irr}\left(\mathcal{H}_{\xi}\right) \mapsto \mathcal{B}^{\text {opt }}, \phi \mapsto \chi_{\phi}$ is a bijection.

Hence, if $\mathcal{H}_{q}$ admits an optimal basic set, then all irreducible representations of $\mathcal{H}_{\xi}$ are obtained as modular reductions of irreducible representations of $\mathcal{H}_{q}$ : we say that all irreducible representations of $\mathcal{H}_{\xi}$ can be lifted to $\mathcal{H}_{q}$. Therefore, we can reorder the rows of the decomposition matrix so that its upper part (consisting of the rows indexed by the elements of $\mathcal{B}^{\text {opt }}$ ) is the identity matrix. Moreover, the optimal basic set produces the full decomposition matrix automatically.

In all the cases studied in this paper, we will show that $\mathcal{H}_{q}$ admits an optimal basic set with respect to any specialization $\theta$ (if $\mathcal{H}_{\xi}$ is semisimple, there is nothing to prove). Moreover, we have checked (using GAP) that $\mathcal{H}_{q}$ also admits a canonical basic set with respect to any $\theta$.

\subsection{Block partitions and good central elements}

Let $\theta: \mathbb{C}\left[y, y^{-1}\right] \rightarrow \mathbb{C}, q \mapsto \xi$ be a specialization of $\mathcal{H}_{q}$ as above. We say that $\chi, \psi \in \operatorname{Irr}(W)$ belong to the same block if they label the rows of the same block in the decomposition matrix

$$
\left(d_{\chi, \phi}^{\theta}\right)_{\chi \in \operatorname{Irr}(W), \phi \in \operatorname{Irr}\left(\mathcal{H}_{\xi}\right)} .
$$

If an irreducible character is alone in its block, then we call it a character of defect zero. We have that $\chi$ is an irreducible character of defect zero if and only if $\theta\left(s_{\chi}\right) \neq 0$ (see [25, Lemma 2.6]).

It is well known that $\chi, \psi \in \operatorname{Irr}(W)$ belong to the same block if and only if $\theta\left(\omega_{\chi}(z)\right)=$ $\theta\left(\omega_{\psi}(z)\right)$ for any $z \in Z\left(\mathcal{H}_{q}\right)$, where $\omega_{\chi}, \omega_{\psi}$ denote the corresponding central characters (see $[\mathbf{1 7}$, Lemma 7.5.10]). Unfortunately, any precise description of the centre, as in [18], is not yet known for our cyclotomic Hecke algebras. However, by [8, 4.20, 5.13], we can know the character values of certain 'good' central elements. Here is a list of some good central elements for our complex reflection groups:

\begin{tabular}{c|ccccccc}
\hline Type & $G_{4}$ & $G_{5}$ & $G_{8}$ & $G_{9}$ & $G_{10}$ & $G_{16}$ & $G_{20}$ \\
Good element & $(s t)^{3}$ & $(s t)^{2}$ & $(s t)^{3}$ & $(s t)^{3}$ & $(s t)^{2}$ & $(s t)^{3}$ & $(s t)^{5}$ \\
\hline
\end{tabular}

Let $\chi \in \operatorname{Irr}(W)$. Following the results of Broué and Michel, the value of $\omega_{\chi}$ at these good central elements is of the form

$$
\left.\lambda(\chi):=\exp \left(2 \pi \sqrt{-1}\left(a_{\chi}+A_{\chi}\right) / d^{2}\right)\right) \gamma_{\chi} q^{\left(a_{\chi}+A_{\chi}\right) / d},
$$

where $d$ and $\gamma_{\chi}$ are complex numbers completely determined by $W$. So, we get a necessary condition

$$
\theta(\lambda(\chi))=\theta(\lambda(\psi))
$$


for the block linkage of $\chi, \psi \in \operatorname{Irr}(W)$. Note that this argument is not new: Geck used this method in $[\mathbf{1 3}, 12.4]$ for the case of Weyl groups.

EXAMPLE 3 . Let $W=G_{10}$ and

$$
\mathcal{H}_{q}\left(G_{10}\right)=\left\langle s, t \mid s t s t=t s t s, \begin{array}{l}
(s-1)\left(s+q^{2}\right)\left(s-q^{4}\right)=0 \\
(t-1)\left(t-q^{3}\right)\left(t+q^{3}\right)\left(t-q^{6}\right)=0
\end{array}\right\rangle .
$$

Following the notation of characters in the GAP3 package CHEVIE, each irreducible character of $W$ is denoted by $\phi_{d, b}$, where $d$ is the dimension of the representation and $b$ is the valuation of its fake degree. The value $\lambda\left(\phi_{d, b}\right)$ of $\mathcal{H}\left(G_{10}\right)$ at the central element $(s t)^{2}=(t s)^{2}$ is given by the following table:

$$
\begin{aligned}
& \begin{array}{c|c|cc|cc|cc|cccc}
\phi_{1,0} & \phi_{1,8} & \phi_{2,1} & \phi_{2,4} & \phi_{1,6} & \phi_{1,12} & \phi_{2,5} & \phi_{2,8} & \phi_{2,7}^{\prime} & \phi_{3,2} & \phi_{2,7}^{\prime \prime} & \phi_{3,6}^{\prime} \\
\hline 1 & q^{4} & q^{5} & -q^{5} & q^{6} & q^{6} & -q^{7} & q^{7} & q^{8} & \zeta_{3}^{2} q^{8} & -q^{8} & q^{8}
\end{array} \\
& \begin{array}{cc|cccc|cccccc}
\phi_{1,16} & \phi_{3,10}^{\prime \prime} & \phi_{4,9} & \phi_{4,3} & \phi_{2,9} & \phi_{2,12} & \phi_{2,11}^{\prime \prime} & \phi_{3,4} & \phi_{2,11}^{\prime} & \phi_{3,10}^{\prime} & \phi_{1,14} & \phi_{3,12}^{\prime \prime} \\
\hline q^{8} & \zeta_{3} q^{8} & -q^{9} & q^{9} & q^{9} & -q^{9} & q^{10} & \zeta_{3} q^{10} & -q^{10} & \zeta_{3} q^{10} & q^{10} & q^{10}
\end{array} \\
& \begin{array}{cccccc|cc|cccc}
\phi_{1,20} & \phi_{4,11} & \phi_{3,14} & \phi_{4,5} & \phi_{3,6}^{\prime \prime} & \phi_{3,8}^{\prime} & \phi_{4,13} & \phi_{4,7} & \phi_{2,13} & \phi_{2,10} & \phi_{2,15}^{\prime} & \phi_{3,8}^{\prime \prime} \\
\hline q^{10} & -i q^{10} & \zeta_{3}^{2} q^{10} & i q^{10} & q^{10} & \zeta_{3}^{2} q^{10} & q^{11} & -q^{11} & -q^{11} & q^{11} & q^{12} & \zeta_{3}^{2} q^{12}
\end{array} \\
& \begin{array}{cccc|cc|cc|cc|c|c}
\phi_{3,12}^{\prime} & \phi_{3,16} & \phi_{1,18} & \phi_{2,15}^{\prime \prime} & \phi_{2,14} & \phi_{2,17} & \phi_{1,22} & \phi_{1,28} & \phi_{2,18} & \phi_{2,21} & \phi_{1,26} & \phi_{1,34} \\
\hline q^{12} & \zeta_{3} q^{12} & q^{12} & -q^{12} & -q^{13} & q^{13} & q^{14} & q^{14} & q^{15} & -q^{15} & q^{16} & q^{20}
\end{array}
\end{aligned}
$$

Let $\theta$ be a specialization of $\mathcal{H}_{q}\left(G_{10}\right)$ such that $\theta(q)=\zeta_{7}$. By looking at the Schur elements, we can easily check (with the use of GAP) that all irreducible characters are of defect zero, except for the following ones:

$$
\phi_{1,0}, \phi_{1,12}, \phi_{2,8}, \phi_{2,17}, \phi_{1,28}, \phi_{1,34} \text {. }
$$

According to the above table, we have

$$
\theta\left(\lambda\left(\phi_{1,0}\right)\right)=\theta\left(\lambda\left(\phi_{2,8}\right)\right)=\theta\left(\lambda\left(\phi_{1,28}\right)\right)=1
$$

and

$$
\theta\left(\lambda\left(\phi_{1,12}\right)\right)=\theta\left(\lambda\left(\phi_{2,17}\right)\right)=\theta\left(\lambda\left(\phi_{1,34}\right)\right)=\zeta_{7}^{6}
$$

Due to the necessary condition for block linkage given above, we must have two separate blocks consisting of three characters each, namely

$$
B_{1}=\left\{\phi_{1,0}, \phi_{2,8}, \phi_{1,28}\right\} \quad \text { and } \quad B_{2}=\left\{\phi_{1,12}, \phi_{2,17}, \phi_{1,34}\right\} .
$$

\section{Methodology and results}

\subsection{Summarized main results and sketch of the proof}

Let $\mathcal{H}_{q}(W)$ be one of the cyclotomic Hecke algebras in the list of $\S 3.3$. Set $\mathcal{O}:=\mathbb{C}\left[q, q^{-1}\right]$ and let $\theta: \mathcal{O} \rightarrow \mathbb{C}, q \mapsto \xi$ be a ring homomorphism as above.

Theorem 4. There exists an optimal basic set for $\mathcal{H}_{q}(W)$ with respect to any specialization $\theta$. 
We have proved the above result case by case, by calculating the decomposition matrix in all the cases where the algebra $\mathcal{H}_{\xi}$ is not semisimple, that is, all the cases where the decomposition matrix is not the identity matrix. By [17, Theorem 7.4.7], $\mathcal{H}_{\xi}$ is not semisimple if and only if there exists $\chi \in \operatorname{Irr}(W)$ such that $\theta\left(s_{\chi}\right)=0$. Due to the form of the Schur elements, this happens only if $\xi$ is a root of unity. In $\S 3.3$, we give the optimal basic sets in all the cases that need to be considered.

Now, the following criteria have been enough to help us determine the decomposition matrices in all the cases that are of interest to us.

(1) We have $\theta\left(s_{\chi}\right) \neq 0$ if and only if $\chi$ is an irreducible character of defect zero.

(2) All one-dimensional representations are irreducible.

(3) If a representation has one of the one-dimensional subrepresentations found in the previous step, then it is reducible. In particular, two-dimensional representations are irreducible, unless they have a one-dimensional subrepresentation. This can be easily checked, because one-dimensional subrepresentations correspond to one-dimensional eigenspaces for the generating matrices of the two-dimensional representation.

(4) We have the following necessary condition for block linkage (see $\S 2.4)$ : if $\chi, \psi \in \operatorname{Irr}(W)$ are in the same block, then $\theta(\lambda(\chi))=\theta(\lambda(\psi))$.

(5) We apply Lemma 6, taking $P(y)$ to be the least common multiple of all Schur elements of $\mathcal{H}_{q}$.

The above criteria give us in each case an optimal basic set and thus a complete description of the irreducible representations of $\mathcal{H}_{\xi}$. We complete the decomposition matrix by using the fact that the modular reductions of the irreducible characters of $\mathcal{H}_{q}$ can be written uniquely as $\mathbb{N}$-linear combinations of the irreducible characters of $\mathcal{H}_{\xi}$.

The irreducible representations of the cyclotomic Hecke algebras associated with the exceptional complex reflection groups have been calculated by Malle and Michel [24]. We are grateful to them for providing us with preliminary versions of their paper and for implementing everything (generating matrices, character tables, etc.) in the GAP3 package CHEVIE, which has been used for all our calculations.

Now, having computed the decomposition matrix, it is easy to check the validity of the following result.

Theorem 5. There exists a canonical basic set for $\mathcal{H}_{q}(W)$ with respect to any specialization $\theta$.

In the Appendix of this paper, we will work out in detail the example of $G_{12}$ for $\xi=\zeta_{8}$. It is a small, but representative, example of the computational work we had to do to obtain Theorems 4 and 5 , as well as the data presented in $\S 3.3$. It illustrates the method, the CHEVIE commands and the programs used in the process.

\subsection{Decomposition matrix and generic degrees}

Recall that $q=y^{|\mu(K)|}$. Let $\zeta$ be a primitive root of unity of order $m$ and consider a specialization $\theta$ such that $\theta(y)=\zeta$. Let $\mathfrak{n}$ be the ideal of $\mathcal{O}$ generated by the irreducible polynomial $y-\zeta$. Note that $\mathcal{O}_{\mathfrak{n}}$ is a discrete valuation ring.

If $\zeta^{\prime}$ is a primitive root of unity of order $m$ such that $\zeta \neq \zeta^{\prime}$, and $\mathfrak{n}^{\prime}$ is the ideal of $\mathcal{O}$ generated by $y-\zeta^{\prime}$, then the rings $\mathcal{O}_{\mathfrak{n}}$ and $\mathcal{O}_{\mathfrak{n}^{\prime}}$ are isomorphic (see, for example, [9, Proposition 1.1.2]). Since the decomposition map $d^{\theta}$ is actually defined as the decomposition map of the algebra $\mathcal{O}_{\mathfrak{n}} \mathcal{H}_{q}\left[\mathbf{1 7}\right.$, Theorem 7.4.3], the decomposition matrix of $\mathcal{H}_{q}$ with respect 
to $\theta$ is the 'same' $\dagger$ as the decomposition matrix with respect to $\theta^{\prime}: y \mapsto \zeta^{\prime}$. Hence, the form of the decomposition matrix does not depend on the choice of the root of unity $\zeta$, but on its order $m$.

Let $\left(d_{\chi, \phi}^{\theta}\right)_{\chi \in \operatorname{Irr}(W), \phi \in \operatorname{Irr}\left(\mathcal{H}_{\xi}\right)}$ be the decomposition matrix of $\mathcal{H}_{q}$ with respect to $\theta$. By $[\mathbf{1 8}$, Proposition 4.4], for all $\phi \in \operatorname{Irr}\left(\mathcal{H}_{\xi}\right)$, we must have

$$
\sum_{\chi \in \operatorname{Irr}(W)} \frac{d_{\chi, \phi}^{\theta}}{s_{\chi}} \in \mathcal{O}_{\mathfrak{n}} .
$$

If $P(y) \in K\left[y, y^{-1}\right]$ is a product of $K$-cyclotomic polynomials which is divisible by all Schur elements of $\mathcal{H}_{q}$, then, for all $\phi \in \operatorname{Irr}\left(\mathcal{H}_{\xi}\right)$, we obtain that

$$
\sum_{\chi \in \operatorname{Irr}(W)} \frac{d_{\chi, \phi}^{\theta} D_{\chi}^{P}}{P(y)} \in \mathcal{O}_{\mathfrak{n}} .
$$

We deduce that the polynomial

$$
\sum_{\chi \in \operatorname{Irr}(W)} d_{\chi, \phi}^{\theta} D_{\chi}^{P}
$$

has at least as many factors of the form $(y-\zeta)$ as $P(y)$. In particular, the following holds.

Lemma 6. Let $\theta: q \mapsto \xi$ be a specialization of $\mathcal{H}_{q}$ such that $\mathcal{H}_{\xi}$ is not semisimple and let $P(y)$ be an element of $K\left[y, y^{-1}\right]$ as above. If $\zeta^{|\mu(K)|}=\xi$, then, for all $\phi \in \operatorname{Irr}\left(\mathcal{H}_{\xi}\right)$,

$$
\sum_{\chi \in \operatorname{Irr}(W)} d_{\chi, \phi}^{\theta} D_{\chi}^{P}(\zeta)=0
$$

\subsection{Decomposition matrices for the exceptional groups of rank two}

Let $W$ be an exceptional irreducible complex reflection group of rank two. For each cyclotomic specialization appearing in $[4,23]$, we will present here an optimal basic set for $\mathcal{H}_{q}(W)$ with respect to any specialization $q=\xi$ such that $\mathcal{H}_{\xi}$ is not semisimple. The modular reductions of the elements of the optimal basic set are the irreducible characters of $\mathcal{H}_{\xi}$. The actual decomposition matrix can be calculated very easily, since all modular reductions of irreducible characters of $\mathcal{H}_{q}$ are written uniquely as $\mathbb{N}$-linear combinations of the elements of $\operatorname{Irr}\left(\mathcal{H}_{\xi}\right)$.

We will not write down the characters of the basic set whose Schur element does not become zero when $q=\xi$, that is, the characters of defect zero. In the cases where we have more than one optimal basic set (different irreducible characters of $\mathcal{H}_{q}$ have the same irreducible modular reduction), we will choose the representatives with minimal $a$-value. We will also separate the blocks using parentheses.

Now, if $m$ is an odd positive integer and $\xi$ is a primitive root of unity of order $m$, then $-\xi$ is a primitive root of unity of order $2 m$. This is why, in the cases when $\mathcal{H}_{q} \cong \mathcal{H}_{-q}$ (namely the cases (ii), (iv), (v) and (vii) for $G_{8}$ and the cases of $G_{9}, G_{10}, G_{12}$ and $G_{22}$ ), we will only study one of the two specializations.

Finally, we want to record some information on block structures that appear very often. Therefore, we will write:
(i) $\left[\phi_{1}\right]$,
(ii) $\left[\phi_{1}, \phi_{3}\right]$,
(iii) $\left\langle\phi_{1}, \phi_{3}\right\rangle$,
(iv) $\left\{\phi_{1}, \phi_{2}\right\}$,
(v) $\left[\left[\phi_{1}, \phi_{4}, \phi_{6}\right]\right]$

\footnotetext{
${ }^{\dagger}$ The indexing of the rows may be different: characters of the same degree may be interchanged.
} 
if the decomposition matrix of the corresponding block is, respectively, of the form:

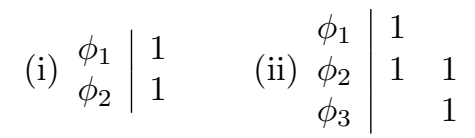

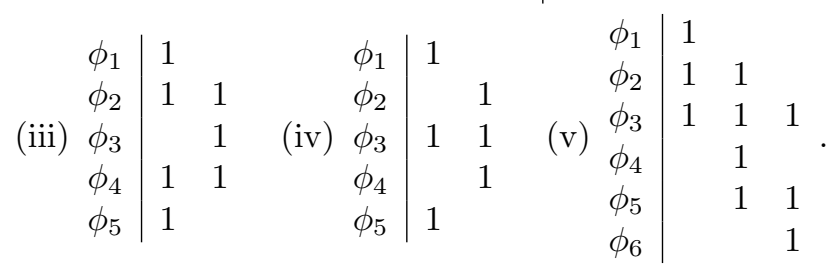

The vacant entry in the decomposition matrices above stands for zero. Actually, in the first two cases, by [28, Theorem 3.6], we know that the block algebras with such decomposition matrices are Brauer tree algebras.

The notation for the characters is the one used by the GAP3 package CHEVIE. The trivial character is always denoted by $\phi_{1,0}$.

3.3.1. Optimal basic sets for the group $G_{4}$.

Let

$$
\mathcal{H}_{q}\left(G_{4}\right)=\left\langle s, t \mid s t s=t s t, \begin{array}{c}
(s-1)(s-q)\left(s-q^{2}\right)=0 \\
(t-1)(t-q)\left(t-q^{2}\right)=0
\end{array}\right\rangle .
$$

The characters that, together with all characters of defect zero, form an optimal basic set for $\mathcal{H}_{q}\left(G_{4}\right)$ are

$$
\begin{aligned}
& q=1:\left(\phi_{1,0}\right),\left(\phi_{2,1}\right) . \\
& q=-1:\left\{\phi_{1,0}, \phi_{2,1}\right\} . \\
& q=\zeta_{6}:\left[\left[\phi_{1,0}, \phi_{1,4}, \phi_{1,8}\right]\right] . \\
& q=\zeta_{12}:\left[\phi_{1,0}, \phi_{1,8}\right] .
\end{aligned}
$$

3.3.2. Optimal basic sets for the group $G_{5}$.

(i) Let

$$
\mathcal{H}_{q}\left(G_{5}\right)=\left\langle s, t \mid s t s t=t s t s, \begin{array}{c}
(s-1)(s-q)\left(s-q^{2}\right)=0, \\
(t-1)(t-q)\left(t-q^{2}\right)=0
\end{array}\right\rangle .
$$

The characters that, together with all characters of defect zero, form an optimal basic set for $\mathcal{H}_{q}\left(G_{5}\right)$ are

$$
\begin{aligned}
& q=1:\left(\phi_{1,0}\right),\left(\phi_{2,1}\right) . \\
& q=-1:\left(\phi_{1,0}, \phi_{1,4^{\prime}}, \phi_{1,4^{\prime \prime}}\right),\left(\phi_{2,1}\right) . \\
& q=i:\left[\left[\phi_{1,0}, \phi_{1,8^{\prime \prime}}, \phi_{1,16}\right]\right],\left[\phi_{1,4^{\prime}}, \phi_{1,12^{\prime}}\right],\left[\phi_{1,4^{\prime \prime}}, \phi_{1,12^{\prime \prime}}\right] . \\
& q=\zeta_{6}:\left[\left[\phi_{1,12^{\prime}}, \phi_{1,0}, \phi_{1,12^{\prime \prime}}\right]\right],\left[\left[\phi_{1,4^{\prime}}, \phi_{1,16}, \phi_{1,4^{\prime \prime}}\right]\right] . \\
& q=\zeta_{8}:\left[\phi_{1,0}, \phi_{1,16}\right] . \\
& q=\zeta_{12}:\left[\phi_{1,0}, \phi_{2,9}\right],\left[\phi_{2,1}, \phi_{1,16}\right] .
\end{aligned}
$$

(ii) Let

$$
\mathcal{H}_{q}\left(G_{5}\right)=\left\langle s, t \mid s t s t=t s t s, \begin{array}{c}
(s-1)(s-q)\left(s-q^{2}\right)=0 \\
(t-1)\left(t-q^{2}\right)\left(t-q^{4}\right)=0
\end{array}\right\rangle
$$


The characters that, together with all characters of defect zero, form an optimal basic set for $\mathcal{H}_{q}\left(G_{5}\right)$ are

$$
\begin{aligned}
& q=1:\left(\phi_{1,0}\right),\left(\phi_{2,1}\right) . \\
& q=-1:\left(\phi_{1,0}, \phi_{1,4^{\prime \prime}}\right),\left(\phi_{2,3^{\prime}}\right) . \\
& q=i:\left\{\phi_{1,0}, \phi_{1,8^{\prime \prime \prime}}\right\},\left[\phi_{2,1}\right],\left\{\phi_{2,3^{\prime}}, \phi_{1,4^{\prime \prime}}\right\},\left[\phi_{2,5^{\prime}}\right] . \\
& q=\zeta_{6}:\left[\left[\phi_{1,0}, \phi_{1,8^{\prime \prime}}, \phi_{1,16}\right]\right],\left[\left[\phi_{1,4^{\prime}}, \phi_{1,12^{\prime}}, \phi_{1,8^{\prime \prime}}\right]\right],\left[\left[\phi_{1,8^{\prime}}, \phi_{1,4^{\prime \prime}}, \phi_{1,12^{\prime \prime}}\right]\right] . \\
& q=\zeta_{8}:\left[\phi_{1,0}, \phi_{1,12^{\prime \prime}}\right],\left[\phi_{1,4^{\prime}}, \phi_{1,16}\right] . \\
& q=\zeta_{10}:\left[\phi_{1,0}, \phi_{1,12^{\prime}}\right],\left[\phi_{1,4^{\prime \prime}}, \phi_{1,16}\right] . \\
& q=\zeta_{12}:\left[\phi_{1,0}, \phi_{1,16}\right],\left[\phi_{1,4^{\prime \prime}}, \phi_{2,7^{\prime \prime}}\right],\left[\phi_{1,12^{\prime}}, \phi_{2,3^{\prime}}\right] . \\
& q=\zeta_{18}:\left[\phi_{1,0}, \phi_{2,9}\right],\left[\phi_{2,1}, \phi_{1,16}\right] .
\end{aligned}
$$

(iii) Let

$$
\mathcal{H}_{q}\left(G_{5}\right)=\left\langle s, t \mid s t s t=t s t s, \begin{array}{c}
(s-1)(s-q)\left(s-q^{2}\right)=0 \\
(t-1)\left(t-q^{4}\right)\left(t-q^{8}\right)=0
\end{array}\right\rangle .
$$

The characters that, together with all characters of defect zero, form an optimal basic set for $\mathcal{H}_{q}\left(G_{5}\right)$ are

$$
\begin{aligned}
& q=1:\left(\phi_{1,0}\right),\left(\phi_{2,1}\right) . \\
& q=-1:\left(\phi_{1,0}, \phi_{1,4^{\prime \prime}}\right),\left(\phi_{2,3^{\prime}}\right) . \\
& q=i:\left(\phi_{1,0}, \phi_{1,8^{\prime \prime \prime}}\right),\left(\phi_{1,4^{\prime \prime}}\right),\left(\phi_{2,1}\right),\left(\phi_{2,5^{\prime}}\right) . \\
& q=\zeta_{6}:\left[\left[\phi_{1,0}, \phi_{1,12^{\prime}}, \phi_{1,12^{\prime \prime}}\right]\right],\left[\left[\phi_{1,8^{\prime}}, \phi_{1,8^{\prime \prime}}, \phi_{1,8^{\prime \prime}}\right]\right],\left[\left[\phi_{1,4^{\prime}}, \phi_{1,4^{\prime \prime}}, \phi_{1,16}\right]\right] . \\
& q=\zeta_{8}:\left[\phi_{1,0}\right],\left\{\phi_{1,4^{\prime \prime}}, \phi_{2,3^{\prime}}\right\},\left[\phi_{2,1}\right],\left[\phi_{1,8^{\prime \prime \prime}}\right],\left[\phi_{2,5^{\prime}}\right] . \\
& q=\zeta_{10}:\left[\left[\phi_{1,0}, \phi_{1,8^{\prime \prime}}, \phi_{1,16}\right]\right],\left[\phi_{1,4^{\prime}}, \phi_{1,12^{\prime}}\right],\left[\phi_{1,4^{\prime \prime}}, \phi_{1,12^{\prime \prime}}\right] . \\
& q=\zeta_{12}:\left[\phi_{1,0}, \phi_{1,12^{\prime \prime}}\right],\left[\phi_{1,8^{\prime}}, \phi_{1,8^{\prime \prime \prime}}\right],\left[\phi_{1,16}, \phi_{1,4^{\prime}}\right] . \\
& q=\zeta_{14}:\left[\phi_{1,8^{\prime}}, \phi_{1,4^{\prime \prime}}\right],\left[\phi_{1,12^{\prime}}, \phi_{1,8^{\prime \prime}}\right] . \\
& q=\zeta_{18}:\left[\phi_{1,0}, \phi_{1,12^{\prime}}\right],\left[\phi_{1,4^{\prime \prime}}, \phi_{1,16}\right],\left[\phi_{1,8^{\prime}}, \phi_{2,5^{\prime}}\right],\left[\phi_{1,8^{\prime \prime \prime}}, \phi_{2,5^{\prime \prime}}\right] . \\
& q=\zeta_{20}:\left[\phi_{1,0}, \phi_{1,16}\right] . \\
& q=\zeta_{24}:\left[\phi_{2,7^{\prime \prime}}, \phi_{1,4^{\prime \prime}}\right],\left[\phi_{1,12^{\prime}}, \phi_{2,3^{\prime}}\right] . \\
& q=\zeta_{30}:\left[\phi_{1,0}, \phi_{2,9}\right],\left[\phi_{2,1}, \phi_{1,16}\right] .
\end{aligned}
$$

3.3.3. Optimal basic sets for the group $G_{8}$.

(i) Let

$$
\mathcal{H}_{q}\left(G_{8}\right)=\left\langle s, t \mid s t s=t s t, \begin{array}{l}
(s-1)\left(s-\zeta_{8}^{3} q\right)\left(s-\zeta_{8}^{5} q\right)\left(s-q^{2}\right)=0 \\
(t-1)\left(t-\zeta_{8}^{3} q\right)\left(t-\zeta_{8}^{5} q\right)\left(t-q^{2}\right)=0
\end{array}\right\rangle .
$$

The characters that, together with all characters of defect zero, form an optimal basic set for $\mathcal{H}_{q}\left(G_{8}\right)$ are

$$
\begin{aligned}
q & =1:\left\{\phi_{1,0}, \phi_{3,2}\right\},\left[\phi_{2,1}\right],\left[\phi_{2,4}\right] . \\
q & =-1:\left\{\phi_{1,0}, \phi_{3,2}\right\},\left[\phi_{2,1}\right],\left[\phi_{2,4}\right] . \\
q & =i:\left[\left[\phi_{1,0}, \phi_{2,7^{\prime \prime}}, \phi_{1,18}\right]\right] . \\
q & =\zeta_{8}^{3}:\left\langle\phi_{1,0}, \phi_{2,10}\right\rangle,\left\langle\phi_{1,6}, \phi_{2,4}\right\rangle,\left(\phi_{2,1}\right) . \\
q & =\zeta_{8}^{5}:\left\langle\phi_{1,0}, \phi_{2,13}\right\rangle,\left\langle\phi_{1,12}, \phi_{2,1}\right\rangle,\left(\phi_{2,4}\right) . \\
q & =\zeta_{12}:\left[\phi_{1,0}, \phi_{1,18}\right] . \\
q & =\zeta_{24}:\left[\phi_{1,0}, \phi_{2,10}\right],\left[\phi_{2,1}, \phi_{2,13}\right],\left[\phi_{2,4}, \phi_{1,18}\right] .
\end{aligned}
$$


(ii) Let

$$
\mathcal{H}_{q}\left(G_{8}\right)=\left\langle s, t \mid s t s=t s t, \begin{array}{l}
(s-1)(s+q)(s-q)\left(s-q^{2}\right)=0 \\
(t-1)(t+q)(t-q)\left(t-q^{2}\right)=0
\end{array}\right\rangle .
$$

The characters that, together with all characters of defect zero, form an optimal basic set for $\mathcal{H}_{q}\left(G_{8}\right)$ are

$$
\begin{aligned}
& q=-1:\left(\phi_{1,0}, \phi_{2,4}\right),\left(\phi_{2,1}\right) . \\
& q=\zeta_{6}:\left[\left[\phi_{1,0}, \phi_{1,12}, \phi_{1,18}\right]\right] . \\
& q=\zeta_{8}:\left[\phi_{1,0}, \phi_{3,8}\right],\left[\phi_{3,2}, \phi_{1,18}\right] . \\
& q=\zeta_{12}:\left[\phi_{1,0}, \phi_{1,18}\right],\left[\phi_{2,1}, \phi_{2,13}\right],\left[\phi_{2,4}, \phi_{2,10}\right] .
\end{aligned}
$$

(iii) Let

$$
\mathcal{H}_{q}\left(G_{8}\right)=\left\langle s, t \mid s t s=t s t, \begin{array}{l}
(s-1)(s-q)\left(s-q^{2}\right)\left(s-q^{3}\right)=0, \\
(t-1)(t-q)\left(t-q^{2}\right)\left(t-q^{3}\right)=0
\end{array}\right\rangle .
$$

The characters that, together with all characters of defect zero, form an optimal basic set for $\mathcal{H}_{q}\left(G_{8}\right)$ are

$$
\begin{aligned}
& q=1:\left(\phi_{1,0}, \phi_{3,2}\right),\left(\phi_{2,1}\right) . \\
& q=-1:\left(\phi_{1,0}, \phi_{1,6}, \phi_{2,1}\right) . \\
& q=\zeta_{3}:\left\{\phi_{2,1}, \phi_{2,4}\right\},\left\{\phi_{1,0}, \phi_{3,2}\right\} . \\
& q=\zeta_{6}:\left(\phi_{1,0}, \phi_{1,6}, \phi_{1,12}, \phi_{1,18}\right),\left[\phi_{2,4}, \phi_{2,10}\right] . \\
& q=\zeta_{8}:\left[\phi_{1,0}, \phi_{2,10}\right],\left[\phi_{2,4}, \phi_{1,18}\right] . \\
& q=\zeta_{10}:\left[\phi_{1,0}, \phi_{2,13}\right],\left[\phi_{2,1}, \phi_{1,18}\right] . \\
& q=\zeta_{12}:\left[\phi_{1,0}, \phi_{1,12}\right],\left[\phi_{1,6}, \phi_{1,18}\right],\left[\phi_{2,1}, \phi_{2,13}\right] . \\
& q=\zeta_{18}:\left[\phi_{1,0}, \phi_{1,18}\right] .
\end{aligned}
$$

(iv) Let

$$
\mathcal{H}_{q}\left(G_{8}\right)=\left\langle s, t \mid s t s=t s t, \begin{array}{c}
(s-1)(s-q)(s+q)\left(s+q^{4}\right)=0 \\
(t-1)(t-q)(t+q)\left(t+q^{4}\right)=0
\end{array}\right\rangle .
$$

Surprisingly, the Poincaré polynomial is equal to the Schur element associated with $\phi_{1,18}$. The characters that, together with all characters of defect zero, form an optimal basic set for $\mathcal{H}_{q}\left(G_{8}\right)$ are

$$
\begin{aligned}
q & =-1:\left(\phi_{1,0}, \phi_{1,6}, \phi_{2,1}\right) . \\
q & =\zeta_{3}:\left(\phi_{1,0}, \phi_{1,12}, \phi_{2,7^{\prime \prime}}\right),\left[\phi_{2,1}, \phi_{2,13}\right],\left[\phi_{3,2}\right] . \\
q & =\zeta_{5}:\left[\phi_{1,0}, \phi_{2,10}\right],\left[\phi_{3,2}, \phi_{1,18}\right] . \\
q & =\zeta_{7}:\left[\phi_{2,4}, \phi_{1,18}\right] . \\
q & =\zeta_{8}:\left[\phi_{1,0}\right],\left[\phi_{2,1}\right],\left[\phi_{2,4}\right],\left[\phi_{3,2}\right] . \\
q & =\zeta_{9}:\left[\phi_{1,6}, \phi_{1,18}\right] . \\
q & =\zeta_{12}:\left[\phi_{1,0}, \phi_{1,18}\right],\left[\phi_{2,1}, \phi_{2,13}\right],\left[\phi_{2,4}, \phi_{2,10}\right] .
\end{aligned}
$$

(v) Let

$$
\mathcal{H}_{q}\left(G_{8}\right)=\left\langle s, t \mid s t s=t s t, \begin{array}{l}
(s-1)\left(s-q^{3}\right)\left(s+q^{3}\right)\left(s+q^{4}\right)=0 \\
(t-1)\left(t-q^{3}\right)\left(t+q^{3}\right)\left(t+q^{4}\right)=0
\end{array}\right\rangle
$$


Surprisingly in this case also, the Poincaré polynomial is equal to $q^{63} s_{\phi_{1,0}}$. The characters that, together with all characters of defect zero, form an optimal basic set for $\mathcal{H}_{q}\left(G_{8}\right)$ are

$$
\begin{aligned}
q & =-1:\left(\phi_{1,0}, \phi_{1,6}, \phi_{2,1}\right) . \\
q & =\zeta_{3}:\left(\phi_{1,0}, \phi_{1,18}, \phi_{2,4}\right),\left[\phi_{2,1}, \phi_{2,13}\right],\left[\phi_{3,6}\right] . \\
q & =\zeta_{5}:\left[\phi_{1,0}, \phi_{3,8}\right],\left[\phi_{2,4}, \phi_{1,18}\right] . \\
q & =\zeta_{7}:\left[\phi_{1,0}, \phi_{2,10}\right] . \\
q & =\zeta_{8}:\left[\phi_{1,0}\right],\left[\phi_{2,1}\right],\left[\phi_{2,4}\right],\left[\phi_{3,2}\right] . \\
q & =\zeta_{9}:\left[\phi_{1,0}, \phi_{1,12}\right] . \\
q & =\zeta_{12}:\left[\phi_{1,0}, \phi_{1,18}\right],\left[\phi_{2,1}, \phi_{2,13}\right],\left[\phi_{2,4}, \phi_{2,10}\right] .
\end{aligned}
$$

(vi) Let

$$
\mathcal{H}_{q}\left(G_{8}\right)=\left\langle s, t \mid s t s=t s t, \begin{array}{c}
(s-1)(s+1)(s+q)\left(s-q^{5}\right)=0, \\
(t-1)(t+1)(t+q)\left(t-q^{5}\right)=0
\end{array}\right\rangle .
$$

In this case, all Schur elements divide $s_{\phi_{1,18}}$. The characters that, together with all characters of defect zero, form an optimal basic set for $\mathcal{H}_{q}\left(G_{8}\right)$ are

$$
\begin{aligned}
q & = \pm 1:\left(\phi_{1,0}, \phi_{1,6}, \phi_{2,1}\right) . \\
q & =\zeta_{3}:\left(\phi_{1,0}, \phi_{1,6}, \phi_{1,12}, \phi_{1,18}\right),\left[\phi_{2,7^{\prime \prime}}, \phi_{2,7^{\prime}}\right] . \\
q & =\zeta_{5}:\left\{\phi_{1,0}, \phi_{2,1}\right\},\left[\phi_{2,4}\right],\left[\phi_{3,2}\right] . \\
q & =\zeta_{6}:\left(\phi_{1,0}, \phi_{1,6}, \phi_{1,12}, \phi_{1,18}\right),\left[\phi_{2,4}, \phi_{2,10}\right] . \\
q & =\zeta_{7}:\left[\phi_{3,2}, \phi_{1,18}\right] . \\
q & =\zeta_{8}:\left[\phi_{1,12}\right],\left[\phi_{2,4}\right],\left[\phi_{2,7^{\prime \prime}}\right],\left[\phi_{3,2}\right] . \\
q & =\zeta_{9}:\left[\phi_{2,4}, \phi_{1,18}\right],\left[\phi_{2,1}, \phi_{2,13}\right] . \\
q & =\zeta_{10}:\left\{\phi_{1,6}, \phi_{2,1}\right\},\left[\phi_{3,2}\right],\left[\phi_{2,7^{\prime \prime}}\right] . \\
q & =\zeta_{12}:\left[\phi_{1,12}, \phi_{1,18}\right],\left[\phi_{2,4}, \phi_{2,10}\right],\left[\phi_{2,7^{\prime}}, \phi_{2,7^{\prime \prime}}\right] . \\
q & =\zeta_{14}:\left[\phi_{3,2}, \phi_{1,18}\right] . \\
q & =\zeta_{15}:\left[\phi_{1,6}, \phi_{1,18}\right] . \\
q & =\zeta_{18}:\left[\phi_{2,7^{\prime \prime}}, \phi_{1,18}\right],\left[\phi_{2,1}, \phi_{2,13}\right] . \\
q & =\zeta_{30}:\left[\phi_{1,0}, \phi_{1,18}\right] .
\end{aligned}
$$

(vii) Let

$$
\mathcal{H}_{q}\left(G_{8}\right)=\left\langle s, t \mid s t s=t s t, \begin{array}{l}
(s-1)\left(s+q^{4}\right)\left(s-q^{5}\right)\left(s+q^{5}\right)=0 \\
(t-1)\left(t+q^{4}\right)\left(t-q^{5}\right)\left(t+q^{5}\right)=0
\end{array}\right\rangle .
$$

In this case, all Schur elements divide $s_{\phi_{1,0}}$. The characters that, together with all characters of defect zero, form an optimal basic set for $\mathcal{H}_{q}\left(G_{8}\right)$ are

$$
\begin{aligned}
& q=-1:\left(\phi_{1,0}, \phi_{1,6}, \phi_{2,1}\right) . \\
& q=\zeta_{3}:\left(\phi_{1,0}, \phi_{1,6}, \phi_{1,12}, \phi_{1,18}\right),\left[\phi_{2,4}, \phi_{2,10}\right] . \\
& q=\zeta_{5}:\left\{\phi_{1,0}, \phi_{2,7^{\prime}}\right\},\left[\phi_{2,1}\right],\left[\phi_{3,4}\right] . \\
& q=\zeta_{7}:\left[\phi_{1,0}, \phi_{3,8}\right] . \\
& q=\zeta_{8}:\left[\phi_{1,0}\right],\left[\phi_{2,4}\right],\left[\phi_{2,7^{\prime}}\right],\left[\phi_{3,6}\right] . \\
& q=\zeta_{9}:\left[\phi_{1,0}, \phi_{2,7^{\prime \prime}}\right],\left[\phi_{2,1}, \phi_{2,13}\right] . \\
& q=\zeta_{12}:\left[\phi_{1,0}, \phi_{1,6}\right],\left[\phi_{2,4}, \phi_{2,10}\right],\left[\phi_{2,7^{\prime}}, \phi_{2,7^{\prime \prime}}\right] . \\
& q=\zeta_{15}:\left[\phi_{1,0}, \phi_{1,18}\right] .
\end{aligned}
$$


(viii) Let

$$
\mathcal{H}_{q}\left(G_{8}\right)=\left\langle s, t \mid s t s=t s t, \begin{array}{l}
(s-1)(s+q)\left(s+q^{4}\right)\left(s-q^{5}\right)=0 \\
(t-1)(t+q)\left(t+q^{4}\right)\left(t-q^{5}\right)=0
\end{array}\right\rangle .
$$

The characters that, together with all characters of defect zero, form an optimal basic set for $\mathcal{H}_{q}\left(G_{8}\right)$ are

$$
\begin{aligned}
& q= \pm 1:\left(\phi_{1,0}, \phi_{1,6}, \phi_{2,1}\right) . \\
& q=\zeta_{3}:\left(\phi_{1,0}, \phi_{1,6}, \phi_{1,18}\right) . \\
& q=\zeta_{5}:\left\{\phi_{1,0}, \phi_{3,2}\right\},\left[\phi_{2,1}\right],\left[\phi_{2,4}\right] . \\
& q=\zeta_{6}:\left(\phi_{1,0}, \phi_{1,6}, \phi_{1,12}, \phi_{1,18}\right),\left[\phi_{2,1}, \phi_{2,13}\right] . \\
& q=\zeta_{7}:\left[\phi_{1,6}, \phi_{2,13}\right],\left[\phi_{1,12}, \phi_{2,1}\right] . \\
& q=\zeta_{8}:\left[\phi_{1,0}\right],\left[\phi_{1,6}\right],\left[\phi_{3,2}\right],\left[\phi_{3,4}\right],\left(\phi_{2,1}\right) . \\
& q=\zeta_{9}:\left[\phi_{1,0}, \phi_{2,13}\right],\left[\phi_{2,1}, \phi_{1,18}\right] . \\
& q=\zeta_{10}:\left[\left[\phi_{1,0}, \phi_{2,7^{\prime \prime}}, \phi_{1,18}\right]\right] . \\
& q=\zeta_{12}:\left[\phi_{1,0}, \phi_{1,12}\right],\left[\phi_{2,1}, \phi_{2,13}\right],\left[\phi_{1,6}, \phi_{1,18}\right] . \\
& q=\zeta_{18}:\left[\phi_{1,6}, \phi_{1,12}\right] . \\
& q=\zeta_{24}:\left[\phi_{2,1}, \phi_{2,13}\right] . \\
& q=\zeta_{30}:\left[\phi_{1,0}, \phi_{1,18}\right] .
\end{aligned}
$$

3.3.4. Optimal basic sets for the group $G_{9}$.

Let

$$
\mathcal{H}_{q}\left(G_{9}\right)=\left\langle s, t \mid s t s t s t=t s t s t s, \begin{array}{l}
(s-1)\left(s-q^{4}\right)=0, \\
(t-1)\left(t-q^{2}\right)\left(t-q^{4}\right)\left(t-q^{6}\right)=0
\end{array}\right\rangle .
$$

The characters that, together with all characters of defect zero, form an optimal basic set for $\mathcal{H}_{q}\left(G_{9}\right)$ are

$$
\begin{aligned}
q & =-1:\left(\phi_{1,0}, \phi_{2,5}\right),\left(\phi_{2,1}\right) . \\
q & =\zeta_{3}:\left(\phi_{1,0}, \phi_{1,6}, \phi_{1,12^{\prime}}, \phi_{1,24}\right),\left\{\phi_{2,5}, \phi_{2,4}\right\},\left[\phi_{1,12^{\prime \prime}}, \phi_{1,18^{\prime}}\right],\left[\phi_{3,2}\right],\left[\phi_{3,6^{\prime}}\right] . \\
q & =i:\left(\phi_{1,0}, \phi_{2,10}\right),\left(\phi_{1,6}, \phi_{2,4}\right),\left(\phi_{2,1}\right),\left(\phi_{2,5}\right) . \\
q & =\zeta_{5}:\left[\left[\phi_{1,0}, \phi_{2,11^{\prime \prime}}, \phi_{1,30}\right]\right],\left[\phi_{1,6}, \phi_{2,8}\right],\left[\phi_{2,1}, \phi_{1,24}\right] . \\
q & =\zeta_{7}:\left[\phi_{1,0}, \phi_{2,8}\right],\left[\phi_{2,1}, \phi_{1,30}\right] . \\
q & =\zeta_{9}:\left[\phi_{1,0}, \phi_{1,18^{\prime}}\right],\left[\phi_{1,6}, \phi_{1,24}\right],\left[\phi_{1,12^{\prime \prime}}, \phi_{1,30}\right] . \\
q & =\zeta_{12}:\left[\left[\phi_{2,10}, \phi_{1,0}, \phi_{1,24}\right]\right],\left[\left[\phi_{1,6}, \phi_{1,30}, \phi_{2,4}\right]\right],\left[\phi_{2,1}, \phi_{2,8}\right],\left[\phi_{2,5}, \phi_{2,13}\right] . \\
q & =\zeta_{15}:\left[\phi_{1,0}, \phi_{1,30}\right] . \\
q & =\zeta_{20}:\left[\phi_{1,0}, \phi_{3,12}\right],\left[\phi_{3,2}, \phi_{1,30}\right] . \\
q & =\zeta_{24}:\left[\phi_{1,0}, \phi_{1,24}\right],\left[\phi_{1,6}, \phi_{1,30}\right],\left[\phi_{2,1}, \phi_{2,13}\right],\left[\phi_{2,5}, \phi_{2,8}\right] .
\end{aligned}
$$

3.3.5. Optimal basic sets for the group $G_{10}$.

Let

$$
\mathcal{H}_{q}\left(G_{10}\right)=\left\langle s, t \mid s t s t=t s t s, \begin{array}{l}
(s-1)\left(s+q^{2}\right)\left(s-q^{4}\right)=0, \\
(t-1)\left(t-q^{3}\right)\left(t+q^{3}\right)\left(t-q^{6}\right)=0
\end{array}\right\rangle .
$$

The characters that, together with all characters of defect zero, form an optimal basic set for $\mathcal{H}_{q}\left(G_{10}\right)$ are

$$
\begin{aligned}
& q=-1:\left(\phi_{1,0}, \phi_{1,6}, \phi_{1,8}\right),\left(\phi_{2,1}, \phi_{2,8}\right),\left(\phi_{3,2}\right),\left(\phi_{3,10^{\prime \prime}}\right) . \\
& q=\zeta_{3}:\left(\phi_{1,0}, \phi_{1,12}, \phi_{2,9}\right),\left(\phi_{1,8}, \phi_{2,8}\right),\left(\phi_{2,12}\right),\left(\phi_{2,5}\right),\left(\phi_{2,4}\right),\left(\phi_{1,16}, \phi_{1,28}, \phi_{2,1}\right) .
\end{aligned}
$$




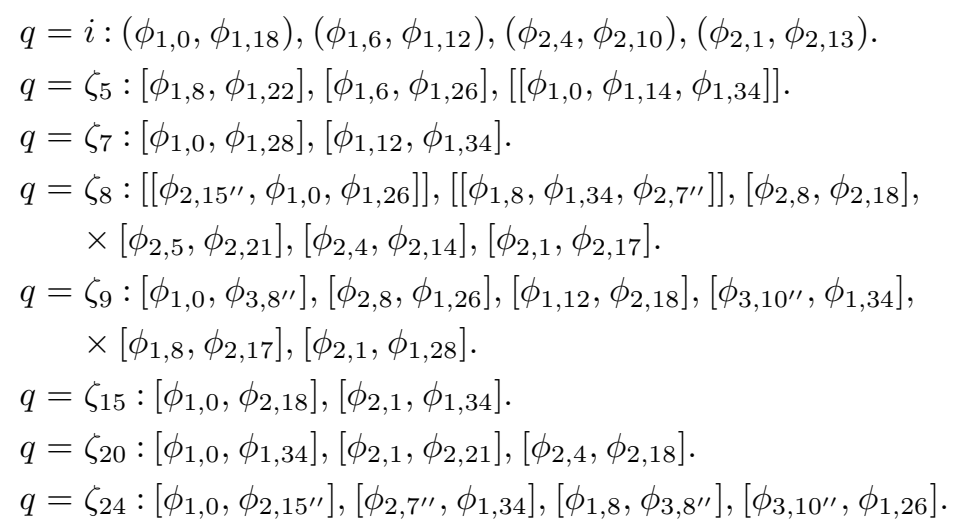

3.3.6. Optimal basic sets for the group $G_{12}$.

Let

$$
\mathcal{H}_{q}\left(G_{12}\right)=\left\langle s, t, u \mid s t u s=t u s t=u s t u, \begin{array}{c}
(s-1)\left(s-q^{2}\right)=0 \\
(t-1)\left(t-q^{2}\right)=0 \\
(u-1)\left(u-q^{2}\right)=0
\end{array}\right\rangle .
$$

The characters that, together with all characters of defect zero, form an optimal basic set for $\mathcal{H}_{q}\left(G_{12}\right)$ are

$$
\begin{aligned}
q & =-1:\left\langle\phi_{1,0}, \phi_{2,4}\right\rangle . \\
q & =\zeta_{8}:\left[\left[\phi_{1,0}, \phi_{1,12}, \phi_{2,1}\right]\right] . \\
q & =\zeta_{12}:\left[\phi_{1,0}, \phi_{1,12}\right] . \\
q & =\zeta_{24}:\left[\phi_{1,0}, \phi_{1,12}\right] .
\end{aligned}
$$

3.3.7. Optimal basic sets for the group $G_{16}$.

Let

$$
\mathcal{H}_{q}\left(G_{16}\right)=\left\langle s, t \mid s t s=t s t, \begin{array}{l}
(s-1)(s-q)\left(s-q^{2}\right)\left(s-q^{3}\right)\left(s-q^{4}\right)=0, \\
(t-1)(t-q)\left(t-q^{2}\right)\left(t-q^{3}\right)\left(t-q^{4}\right)=0
\end{array}\right\rangle .
$$

The characters that, together with all characters of defect zero, form an optimal basic set for $\mathcal{H}_{q}\left(G_{16}\right)$ are

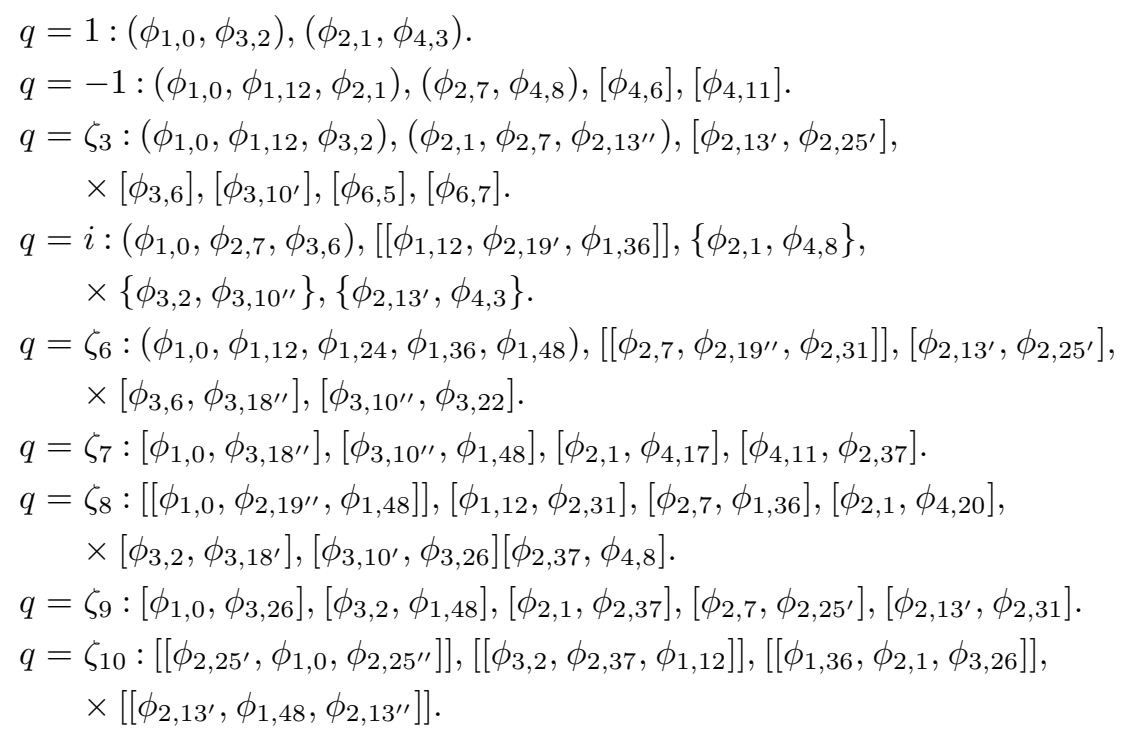




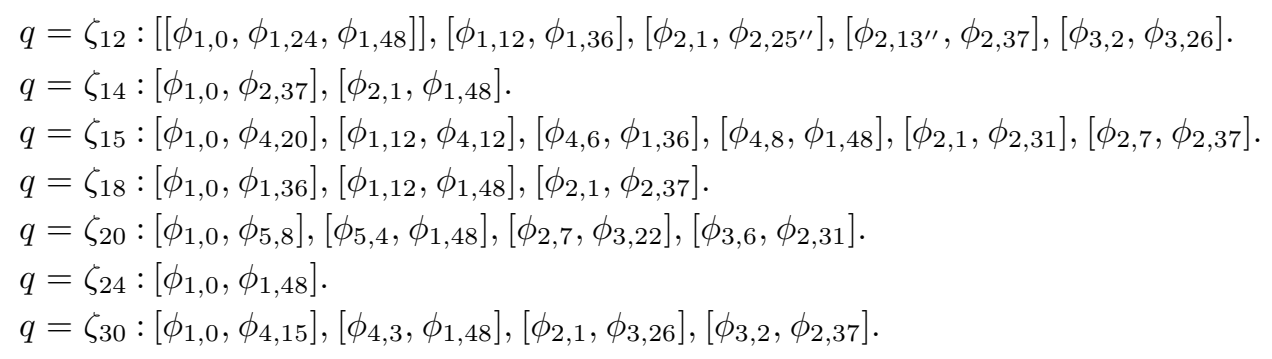

3.3.8. Optimal basic sets for the group $G_{20}$.

Let

$$
\mathcal{H}_{q}\left(G_{20}\right)=\left\langle s, t \mid s t s t s=t s t s t, \begin{array}{c}
(s-1)(s-q)\left(s-q^{2}\right)=0, \\
(t-1)(t-q)\left(t-q^{2}\right)=0
\end{array}\right\rangle .
$$

The characters that, together with all characters of defect zero, form an optimal basic set for $\mathcal{H}_{q}\left(G_{20}\right)$ are

$$
\begin{aligned}
& q=1:\left(\phi_{1,0}, \phi_{4,6}\right),\left(\phi_{2,1}, \phi_{2,7}\right) . \\
& q=-1:\left(\phi_{1,0}, \phi_{2,1}, \phi_{2,7}\right),\left[\phi_{4,3}\right],\left[\phi_{4,6}\right] . \\
& q=i:\left[\left[\phi_{1,0}, \phi_{4,11}, \phi_{1,40}\right]\right] . \\
& q=\zeta_{5}:\left[\left[\phi_{1,0}, \phi_{3,10^{\prime}}, \phi_{1,40}\right]\right],\left[\left[\phi_{2,1}, \phi_{2,11}, \phi_{2,21}\right]\right],\left[\phi_{2,7}, \phi_{2,27}\right] . \\
& q=\zeta_{6}:\left[\left[\phi_{2,21}, \phi_{1,0}, \phi_{2,27}\right]\right],\left[\left[\phi_{2,1}, \phi_{1,40}, \phi_{2,7}\right]\right] . \\
& q=\zeta_{10}:\left[\left[\phi_{1,0}, \phi_{1,20}, \phi_{1,40}\right]\right],\left[\phi_{2,1}, \phi_{2,21}\right] . \\
& q=\zeta_{12}:\left[\phi_{1,0}, \phi_{5,12}\right],\left[\phi_{5,4}, \phi_{1,40}\right] . \\
& q=\zeta_{15}:\left[\phi_{1,0}, \phi_{3,2}\right],\left[\phi_{2,7}, \phi_{4,13}\right],\left[\phi_{4,3}, \phi_{2,27}\right],\left[\phi_{1,40}, \phi_{3,12}\right] . \\
& q=\zeta_{20}:\left[\phi_{1,0}, \phi_{1,40}\right] . \\
& q=\zeta_{30}:\left[\phi_{1,0}, \phi_{2,21}\right],\left[\phi_{1,40}, \phi_{2,1}\right] .
\end{aligned}
$$

3.3.9. Optimal basic sets for the group $G_{22}$.

Let

$$
\left.\mathcal{H}_{q}\left(G_{22}\right)=\langle s, t, u| \text { stust }=\text { tustu }=\text { ustus }, \begin{array}{c}
(s-1)\left(s-q^{2}\right)=0 \\
(t-1)\left(t-q^{2}\right)=0 \\
(u-1)\left(u-q^{2}\right)=0
\end{array}\right\rangle .
$$

The characters that, together with all characters of defect zero, form an optimal basic set for $\mathcal{H}_{q}\left(G_{22}\right)$ are

$$
\begin{aligned}
& q=-1:\left(\phi_{1,0}, \phi_{2,13}, \phi_{2,1}\right) . \\
& q=\zeta_{6}:\left[\left[\phi_{1,0}, \phi_{4,6}, \phi_{1,30}\right]\right] . \\
& q=\zeta_{10}:\left[\left[\phi_{1,0}, \phi_{2,13}, \phi_{1,30}\right]\right],\left[\phi_{3,2}, \phi_{3,12}\right] . \\
& q=\zeta_{12}:\left[\phi_{1,0}, \phi_{4,3}\right],\left[\phi_{4,9}, \phi_{1,30}\right] . \\
& q=\zeta_{20}:\left[\phi_{1,0}, \phi_{3,12}\right],\left[\phi_{3,2}, \phi_{1,30}\right] . \\
& q=\zeta_{30}:\left[\phi_{1,0}, \phi_{1,30}\right] .
\end{aligned}
$$

\subsection{Conjectures and remarks}

The following two conjectures have been checked to hold in all the cases presented in $\S 3.3$. Moreover, Conjecture 8 has been proved for type $A$ and all exceptional Weyl groups in [11], using respectively, $[3,3.7$ and 4.5$]$ and the GAP3 package CHEVIE ${ }^{\dagger}$.

\footnotetext{
${ }^{\dagger}$ For types $A, B$, etc., M. Fayers sent to the second author a sketch of a proof for Conjecture 8 using purely combinatorics.
} 
Let $W$ be a complex reflection group. Let $\theta: q \mapsto \xi$ be a specialization of $\mathcal{H}_{q}(W)$ and let $\left(d_{\chi, \phi}^{\theta}\right)_{\chi \in \operatorname{Irr}(W), \phi \in \operatorname{Irr}\left(\mathcal{H}_{\xi}\right)}$ be the corresponding decomposition matrix.

Conjecture 7. For all $\phi \in \operatorname{Irr}\left(\mathcal{H}_{\xi}\right)$, we have

$$
\theta\left(\sum_{\chi \in \operatorname{Irr}(W)} \frac{d_{\chi, \phi}^{\theta}}{s_{\chi}}\right) \neq 0,
$$

that is, with the notation of Lemma $6, \sum_{\chi \in \operatorname{Irr}(W)} d_{\chi, \phi}^{\theta} D_{\chi}^{P}$ has as many factors of the form $(q-\xi)$ as $P(y)$.

Conjecture 8. If $\chi, \psi \in \operatorname{Irr}(W)$ belong to the same block, then ${ }^{\dagger}$

$$
\theta\left(s_{\chi} / s_{\psi}\right) \in \mathbb{R}^{\times} .
$$

We would like to call the above ratio $\theta\left(s_{\chi} / s_{\psi}\right)$ a Broué invariant. The reason why we call it this is that in [3, 3.7 and 4.5] Broué has shown that these ratios are Morita (respectively derived) invariants (respectively up to sign) for block algebras with a suitable modular system ${ }^{\ddagger}$.

The authors expect that the parity condition on $\operatorname{Ext}^{1}$ for an appropriate quasihereditary cover, namely $\mathcal{O}=\mathcal{O}_{\mathbf{c}}(W, V)$ of the rational Cherednik algebra (see [21] for the definition of the rational Cherednik algebra and $\mathcal{O}$ ), holds. Here, $V$ is the natural representation of $W$ and c is a charge for the rational Cherednik algebra that is consistent with the parameters of the Hecke algebra $\mathcal{H}_{\xi}$ via the KZ-functor.

More precisely, let $\mathcal{B}$ be a block of $\operatorname{Irr}(W)$, which is defined with respect to the specialization $\theta: q \mapsto \xi$. Let $\chi_{\mathcal{B}}$ be the element in $\mathcal{B}$ with minimal $a$-value, which is conjecturally unique ${ }^{\S}$. Conjecture 8 allows us to define a partition $\mathcal{B}=\mathcal{B}_{+} \sqcup \mathcal{B}_{-}$, where

$$
\mathcal{B}_{+}:=\left\{\chi \in \mathcal{B} \mid \theta\left(s_{\chi_{\mathcal{B}}} / s_{\chi}\right)>0\right\} \quad \text { and } \quad \mathcal{B}_{-}:=\left\{\chi \in \mathcal{B} \mid \theta\left(s_{\chi_{\mathcal{B}}} / s_{\chi}\right)<0\right\}
$$

We denote by $L(\lambda)$ the simple module in $\mathcal{O}$ associated with $\lambda \in \operatorname{Irr}(W)$. Define the length function modulo 2 , denoted by $\ell_{\bmod 2}$, on $\operatorname{Irr}(W)$ by

$$
\ell_{\bmod 2}(\lambda):= \begin{cases}0 \in \mathbb{F}_{2} & \text { if } \lambda \in \mathcal{B}_{+} \\ 1 \in \mathbb{F}_{2} & \text { if } \lambda \in \mathcal{B}_{-}\end{cases}
$$

for some block $\mathcal{B} \ni \lambda$. We can state the Parity conjecture, that is, for any $\lambda, \mu \in \operatorname{Irr}(W)$, we have $\operatorname{Ext}_{\mathcal{O}}^{1}(L(\lambda), L(\mu)) \neq\{0\}$ only if

$$
\ell_{\bmod 2}(\lambda) \neq \ell_{\bmod 2}(\mu) \text { and } \lambda \text { and } \mu \text { are in the same block }{ }^{\|} .
$$

Finally, we want to make the following remark on the lifting argument: let $p$ be a prime number. The classical Fong-Swan theorem says that if a group $G$ is $p$-solvable, then all the

\footnotetext{
${ }^{\dagger}$ In fact, in all the examples we have, the ratio belongs to $\mathbb{Q}^{\times}$.

${ }^{\ddagger}$ In [3, 3.7 and 4.5], M. Broué considered the case of group algebras, but his argument is also valid for Hecke algebras. Here, the ratio $\theta\left(s_{\chi} / s_{\psi}\right)$ is slightly different from Broué's original definition, but the interpretation between his and ours is clear if we treat Broué's original ratios in [3] as specialized Schur element ratios and Conjecture 8 is true. The second author would like to thank M. Broué for explaining the proof of $[\mathbf{3}, 3.7$ and 4.5] to him.

$\S$ As in [15], if $W$ is real, this uniqueness is true.

IFor type $A$, this length function is consistent with the Cline-Parshall-Scott length function in Lusztig's conjecture as well as in the abstract Kazhdan-Lusztig theory. So, we expect that the length function we define will be useful for the existence of the strong Kazhdan-Lusztig theory for category $\mathcal{O}$ of rational Cherednik algebras in the sense of Cline-Parshall-Scott.

$\|$ The validity of Lusztig's conjecture for quantum general linear groups at roots of unity and the equivalences between module categories of quantized Schur algebras and the category $\mathcal{O}$ of rational Cherednik algebras of type $A$ yield that the Parity conjecture is true for type $A$.
} 
simple $\mathbb{F}_{p^{k}}[G]$-modules are lifted for some $k>0$ (cf. $\left.[\mathbf{2 6}, 7.5]\right)$. Note that all complex reflection groups that we deal with in this paper, except for the groups $G_{16}, G_{20}$ and $G_{22}$, are solvable. So, if the modular system with parameters is well defined for our specialized Hecke algebra $\mathcal{H}_{\xi}$ in characteristic zero and a group algebra $\mathbb{F}_{p^{k}}[W]$ in positive characteristic, then the modular simple module over the group algebra $\mathbb{F}_{p^{k}}[W]$ can be lifted to $\mathcal{H}_{\xi}$, hence over the generic Hecke algebra by the commutativity of two different modular systems (see [2, p. 141] for the modular system with parameters). In this paper, we have stronger results. The statement above does not say that all simple $\mathcal{H}_{\xi}$-modules are liftable. It only says that some of the simple $\mathcal{H}_{\xi}$-modules are liftable. However, as one of our main results, it turns out that all simple $\mathcal{H}_{\xi}$-modules for our choices of parameters are liftable.

Acknowledgements. The authors would like to thank M. Broué, G. Malle and J. Michel for fruitful discussions on the topics of this paper. In particular, they are indebted to G. Malle and J. Michel for providing them with preliminary versions of their article [24]. The second author would like to thank I. Gordon for his invitation to the University of Edinburgh. This research was partially supported by the Japanese Ministry of Education, Science, Sports and Culture, Grant-in-Aid for Young Scientists (B), 19740011, 2008, and by the EPSRC grant $\mathrm{EP} / \mathrm{G} 04984 \mathrm{X} / 1$.

\section{Appendix}

Let us work out in detail the example of $G_{12}$, when

$$
\left.\mathcal{H}_{q}\left(G_{12}\right)=\langle s, t, u| s t u s=\text { tust }=\text { ustu }, \begin{array}{c}
(s-1)\left(s-q^{2}\right)=0 \\
(t-1)\left(t-q^{2}\right)=0 \\
(u-1)\left(u-q^{2}\right)=0
\end{array}\right\rangle
$$

and $q=\zeta_{8}$. We will use the program GAP3 and we need the packages CHEVIE and VKCURVE. Everything can be found on Jean Michel's webpage:

We type: http://people.math.jussieu.fr/ ${ }^{\sim}$ jmichel

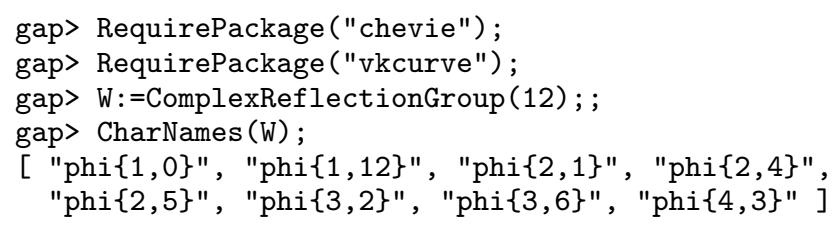

Following the notation of characters in CHEVIE, each irreducible character is denoted by $\phi_{d, b}$, where $d$ is the dimension of the representation and $b$ is the valuation of its fake degree. Therefore, the irreducible characters $\phi_{1,0}$ and $\phi_{1,12}$ are one-dimensional, $\phi_{2,1}, \phi_{2,4}$ and $\phi_{2,5}$ are two-dimensional, $\phi_{3,2}$ and $\phi_{3,6}$ are three-dimensional and $\phi_{4,3}$ is four-dimensional. We now define the algebra $\mathcal{H}_{q}\left(G_{12}\right)$ and check the values of the Schur elements for $q=\zeta_{8}$ :

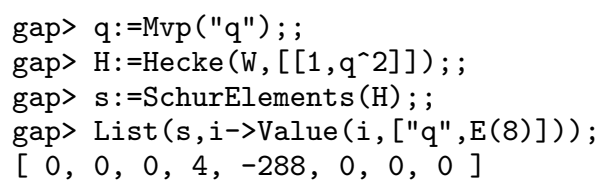

Due to Criterion (1) of $\S 3.1$, the characters $\phi_{2,4}$ and $\phi_{2,5}$ are of defect zero. Now, we will see what happens with the remaining irreducible representations of $\mathcal{H}_{q}\left(G_{12}\right)$ when $q=\zeta_{8}$ :

gap $>\mathrm{R}:=$ Representations $(\mathrm{H}) ;$;

gap> $r:=\operatorname{List}(R, i->\operatorname{List}(i, j->\operatorname{List}(j, k->\operatorname{List}(k, 1->\operatorname{Value}(1,[" q ", E(8)]))))) ;$; 
gap> $r[1]$;

$\left[\left[\left[\begin{array}{ll}1 & ],[\end{array}\right]\right.\right.$

gap> $r[2]$

$\left[\left[\left[I_{1}\right],\left[\left[I_{1}\right],\left[\left[I_{1}\right]\right]\right.\right.\right.$

Hence, the characters $\phi_{1,0}$ and $\phi_{1,12}$ correspond to distinct irreducible representations of $\mathcal{H}_{\zeta_{8}}$. Now, we will check whether the two-dimensional representation $\phi_{2,1}$ has either $\phi_{1,0}$ or $\phi_{1,12}$ as a subrepresentation. For this, it is enough to check whether the generating matrices r[3][1] and r[3][2] have a common eigenvector for either the eigenvalue 1 or $i$. We have created the following (not very sophisticated) functions:

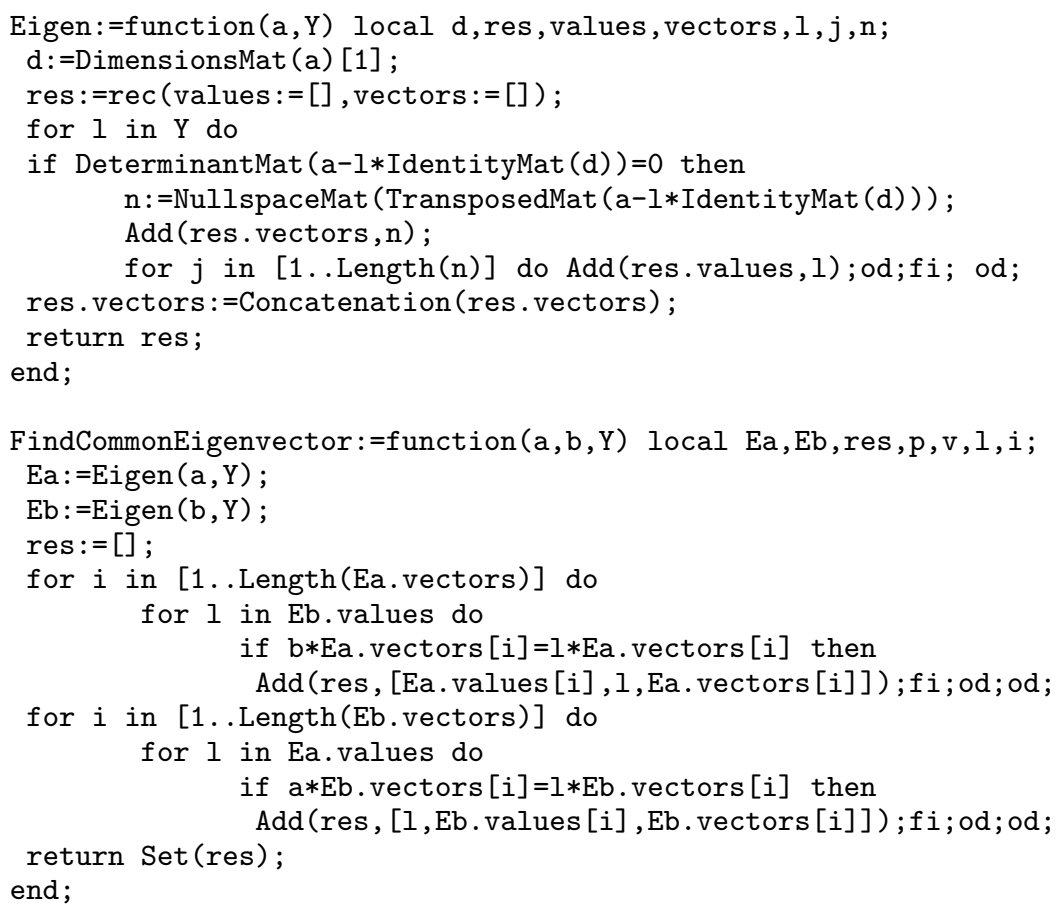

Now, let $a, b$ be two square matrices and $Y$ a list of complex numbers. The function $\operatorname{Eigen}(a, Y)$ returns a record consisting of the elements of $Y$ that are eigenvalues of $a$, and a list of corresponding eigenvectors for $a$. The function FindCommonEigenvector $(a, b, Y)$ returns a list of common eigenvectors for the matrices $a$ and $b$ with corresponding eigenvalues in $Y$ (it is not necessary that these vectors correspond to the same eigenvalue for $a$ and $b$ ). Thus, we check whether $\mathrm{r}[3][1]$ and $\mathrm{r}[3][2]$ have a common eigenvector for either the eigenvalue 1 or $i$ in the following way:

gap> FindCommonEigenvector $(r[3][1], r[3][2],[1, E(4)])$;

[ ]

They do not, so $\phi_{2,1}$ is also irreducible. We shall now apply Lemma 6 on generic degrees, recalling that, in this case, the Poincaré polynomial $P$ is equal to the Schur element s[1].

gap> D:=List $(\mathrm{s}, \mathrm{i}->\mathrm{s}[1] / \mathrm{i}) ;$;

gap> List (D,i->Value (i, ["q",E(8)]));

$[1,1,4,0,0,-3,-3,2]$

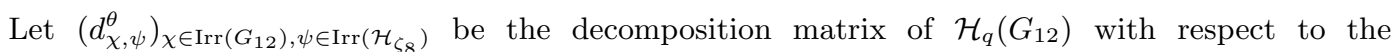
specialization $\theta: q \mapsto \zeta_{8}$. In order to have

$$
\sum_{\chi \in \operatorname{Irr}\left(G_{12}\right)} d_{\chi, \psi}^{\theta} D_{\chi}^{P}\left(\zeta_{8}\right)=0,
$$


the decomposition matrix must look as follows:

\begin{tabular}{l|llllll}
$\phi_{1,0}$ & 1 & & & & \\
$\phi_{3,2}$ & $a$ & 1 & $b$ & & \\
$\phi_{4,3}$ & 1 & 1 & 1 & & \\
$\phi_{2,1}$ & & 1 & & & \\
$\phi_{3,6}$ & $c$ & 1 & $d$ & & \\
$\phi_{1,12}$ & & & 1 & & \\
$\phi_{2,4}$ & & & & 1 & \\
$\phi_{2,5}$ & & & & & 1
\end{tabular}

where $\{(a, b),(c, d)\}=\{(1,0),(0,1)\}$. To find which is which, we use the fact that the modular reductions of the irreducible characters of $\mathcal{H}_{q}\left(G_{12}\right)$ can be written uniquely as $\mathbb{N}$-linear combinations of the irreducible characters of $\mathcal{H}_{\zeta_{8}}$. We type:

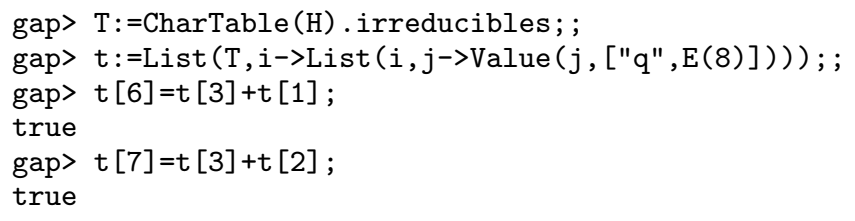

Thus, $(a, b)=(1,0)$ and $(c, d)=(0,1)$, and the decomposition matrix is

\begin{tabular}{l|lllll}
$\phi_{1,0}$ & 1 & & & & \\
$\phi_{3,2}$ & 1 & 1 & & & \\
$\phi_{4,3}$ & 1 & 1 & 1 & & \\
$\phi_{2,1}$ & & 1 & & & \\
$\phi_{3,6}$ & & 1 & 1 & & \\
$\phi_{1,12}$ & & & 1 & & \\
$\phi_{2,4}$ & & & & 1 & \\
$\phi_{2,5}$ & & & & & 1
\end{tabular}

\section{References}

1. S. ARIKI, 'On the decomposition numbers of the Hecke algebra of $G(m, 1, n)$ ', J. Math. Kyoto Univ. 36 (1996) 789-808.

2. S. Ariki and A. Mathas, 'The representation type of Hecke algebras of type B', Adv. Math. 181 (2004) 134-159.

3. M. Broué, 'Equivalences of blocks of group algebras', Finite-dimensional algebras and related topics (Ottawa, ON, 1992), NATO Advanced Science Institutes Series C: Mathematical and Physical Sciences 424 (Kluwer Academic, Dordrecht, 1994) 1-26.

4. M. Broué and G. Malle, 'Zyklotomische Heckealgebren', Astérisque 212 (1993) 119-189.

5. M. Broué, G. Malle and J. Michel, 'Generic blocks of finite reductive groups', Astérisque 212 (1993) $7-92$.

6. M. Broué, G. Malle and J. Michel, 'Towards spetses I', Transform. Groups 4 (1999) no. 2-3, $157-218$.

7. M. Broué, G. Malle and R. Rouquier, 'Complex reflection groups, braid groups, Hecke algebras', J. Reine Angew. Math. 500 (1998) 127-190.

8. M. Broué and J. Michel, 'Sur certains éléments réguliers des groupes de Weyl et les variétés de DeligneLusztig associées', Finite reductive groups (Luminy, 1994), Progress in Mathematics 141 (Birkhäuser, Boston, MA, 1997) 73-139.

9. M. Chlouveraki, Blocks and families for the cyclotomic Hecke algebras, Lecture Notes in Mathematics 1981 (Springer, Berlin, Heidelberg, 2009).

10. M. Chlouveraki and N. JACON, 'Schur elements and basic sets for cyclotomic Hecke algebras', J. Algebra Appl. 10 (2011) 979-993.

11. J. Chunang and H. Miyachi, 'Generic degree ratios at roots of unity', in The 10th Workshop on Representation Theory of Algebraic Groups and Quantum Groups - in honor of Prof. Shoji and Prof. Shinoda's 60th birthdays, S. Ariki et al., Preprint, http://www.math.nagoya-u.ac.jp/ miyachi/articles/raq10pro07.pdf.

12. R. Dipper, G. James and E. Murphy, 'Hecke algebras of type $B_{n}$ at roots of unity', London Math. Soc. 70 (1995) 505-528.

13. M. GECK, 'Brauer trees of Hecke algebras', Comm. Algebra 20 (1992) 2937-2973.

14. M. Geck, Beiträge zur Darstellungstheorie von Iwahori-Hecke-Algebren (Habilitations-schrift, RWTH Aachen, 1993). 
15. M. GEck, Modular representations of Hecke algebras, Group Representation Theory (EPFL Press, Lausanne, 2007) 301-353.

16. M. GECK and N. JACON, 'Canonical basic sets in type $B_{n}$ ', J. Algebra 306 (2006) no. 1, 104-127.

17. M. GeCK and G. Pfeiffer, Characters of Coxeter groups and Iwahori-Hecke algebras, LMS Monographs New Series 21 (Oxford University Press, 2000).

18. M. GECK and R. Rouquier, Centers and simple modules for Iwahori-Hecke algebras, Progress in Mathematics 141 (Birkhäuser, 1997) 251-272.

19. M. GECK and R. Rouquier, 'Filtrations on projective modules for Iwahori-Hecke algebras', Modular representation theory of finite groups (Charlottesville, VA, 1998) (de Gruyter, Berlin, 2001) 211-221.

20. G. Genet and N. JACON, 'Modular representations of cyclotomic Hecke algebras of type $G(r, p, n)$ ', Int. Math. Res. Not. 2006 (2006), doi:10.1155/IMRN/2006/93049.

21. V. Ginzburg, N. Guay, E. Opdam and R. Rouquier, 'On the category $\mathcal{O}$ for rational Cherednik algebras', Invent. Math. 154 (2003) no. 3, 617-651.

22. N. JACON, 'On the parametrization of the simple modules for Ariki-Koike algebras at roots of unity', J. Math. Kyoto Univ. 44 (2004) no. 4, 729-767.

23. G. Malle, Degrés relatifs des algèbres cyclotomiques associées aux groupes de réflexions complexes de dimension deux, Progress in Mathematics 141 (Birkhäuser, 1996) 311-332.

24. G. MAlle and J. Michel, 'Constructing representations of Hecke algebras for complex reflection groups', LMS J. Comput. Math. 13 (2010) 426-450.

25. G. Malle and R. Rouquier, 'Familles de caractères des groupes de réflexions complexes', Represent. Theory 7 (2003) 610-640 (electronic).

26. H. Nagao and Y. Tsushima, Representations of finite groups (Academic Press, Boston, MA, 1989).

27. D. UGLOV, 'Canonical bases of higher-level $q$-deformed Fock spaces and Kazhdan-Lusztig polynomials', Physical combinatorics (Kyoto, 1999), Progress in Mathematics 191 (Birkhäuser, Boston, MA, 2000) 249-299.

28. K. Uno, 'On representations of nonsemisimple specialized Hecke algebras', J. Algebra 149 (1992) no. 2, 287-312.

\author{
Maria Chlouveraki \\ School of Mathematics \\ University of Edinburgh \\ JCMB, Room 5610 \\ King's Buildings \\ Edinburgh EH9 3JZ \\ United Kingdom
}

maria.chlouveraki@ed.ac.uk

\author{
Hyohe Miyachi \\ Graduate School of Mathematics \\ Nagoya University \\ Chikusa-ku, Nagoya Aichi, 464-8602 \\ Japan \\ miyachi@math.nagoya-u.ac.jp
}

